\title{
A Hybrid Top-Down Parsing Technique
}

\author{
HEINZ DOBLER
}

\author{
Universität Linz \\ Institut für Informatik \\ Altenberger Str. 69, 4040 Linz, Austria
}

For top-down parsing two standard methods are widely used: recursive descent and tabledriven analysis. The new method presented in this abstract is called hybrid because it is a sophisticated combination of the two standard methods providing the advantages of both conventional methods while avoiding their disadvantages.

The standard top-down parsing methods mentioned above have their specific benefits and drawbacks:

O Recursive descent parsing allows local semantic objects within each grammar rule. These semantic objects are automatically stacked upon recognition of recursive nonterminals. Parsing speed is maximal, as the recognition of a terminal defaults to a simple comparison instruction. The drawbacks are a grammar-dependent static parser size as well as a lot of code and run-time necessary to implement good error handling and recovery - even when analyzing correct source texts.

O Table-driven parsing offers a small and constant static parser size as the syntax of the source language and the semantic references are coded in a compact data structure (called grammar table). Furthermore, this method allows the implementation of a systematic and grammar-independent error handling, which wastes neither storage nor run-time when parsing correct source texts. The disadvantages are that only global semantic objects can be used, as all semantic actions are collected in a single procedure, and that the compiler implementor has to take care of saving semantic objects on a semantic stack across recursive grammar rules. As the interpretation adds considerable overhead, parsing speed is slow.

This comparison shows a certain duality of both conventional methods and triggered the idea to develop and implement a new hybrid top-down parsing method which provides the advantages of both methods while avoiding all of their disadvantages. The idea of the hybrid method is based on the combination of table-driven syntax analysis and recursive descent semantics evaluation. Therefore a hybrid parser consists of two components:

O The syntax analyzer is a grammar independent driver routine interpreting a grammar table. It does the syntax analysis proper - including error handling and recovery. 
The semantic evaluator is a set of semantic procedures, one for each grammar rule, which contain the declarations of local semantic objects and all the semantic actions for the specific nonterminal. Attributes are represented by formal parameters of the semantic procedures.

The invocation and the termination of these semantic procedures (hence the attribute-passing an the stacking of local semantic objects) as well as the selection of the next semantic action to be executed within the active semantic procedure is dynamically controlled by the driver routine. So the execution of the hybrid parsing method results in a dynamic nesting of the semantic procedures comparable to the procedure nesting in recursive descent parsers.

When a syntax error occurs, the erroneous token and the expected tokens (extracted from the grammar table) are used to generate an error message. The actual position within the grammar table and the list of nonterminals that are currently being analyzed provide sufficient information to collect set of tokens (recovery set) that can be used to continue the syntax analysis. Then a minimal amount of source text is skipped until an element of the recovery set is found and analysis continues at an appropriate position within the grammar table.

While any table-driven method could be adopted to accomplish the special incorporation of semantic evaluation presented here, the new compiler generator system Coco-2 [Dobler90] which implements the hybrid parsing technique - uses an adoption of the proven method developed for Coco [Rechenberg88]. Coco-2 is implemented in Modula-2 on Apple Macintosh computers and generates compilers in Modula-2.

To allow the comparison of hybrid table-driven parsers with hand-coded recursive descent parsers, a recursive descent parser for Modula-2 was implemented and Coco-2 was used to generate a parser for Modula-2. These two parsers were used to analyze a large Modula-2 source text. Measuring pure parsing speed (without $\mathrm{I} / \mathrm{O}$, scanning and semantic evaluation) we found that recursive descent parsing without error handling overhead is about five times faster and recursive descent parsing with error handling overhead is nearly three times faster than the table-driven method. But considering that parsing consumes only a small amount of the runtime of realistic compilers, the run-time penalty of the hybrid method is not of such relevance in practice.

The tests carried out so far fully satisfied our expectations. There are two main areas for further work: The current implementation uses the procedure call mechanism for the communication of the syntax analyzer and the semantic evaluator. Regarding the parse problem as the parallel execution of syntax analysis and semantic evaluation suggests a "parallel" implementation. This can be done e. g. in Modula-2 using co-routines or in Ada with tasks. There is an enormous runtime penalty involved with table interpretation. In favour of a clear and structured interpretation algorithm, up to now, no efforts were made to tune the parser for high speed, so there is a considerable potential for speed-up.

\section{References}

[Dobler90] Dobler H., Pirklbauer K.: Coco-2 - A New Compiler Compiler. SIGPLAN Notices, Vol. 25, May 1990.

[Rechenberg88] Rechenberg P., Mössenböck H.: A Compiler Generator for Microcomputers. Prentice Hall, 1989. 